\title{
Contribución de sistemas productivos en la generación de ingresos en familias cacaoteras, departamento del Caquetá
}

\author{
Income-generating contribution of production systems for cocoa farming families, \\ department of Caqueta
}

\section{Diana María Sánchez Olaya ${ }^{1}$; Oscar Gerardo Velandia Tibáquira ${ }^{2}$; Juan Carlos Suárez Salazar ${ }^{3}$}

1 Docente, I. Agroecólogo. Mg. Grupo de Investigación en Agroecosistemas y Conservación en Bosques Amazónicos, Universidad de la Amazonía. diana-maria26@hotmail.com

2 Técnico, I. Agroecólogo. Grupo de Investigación en Agroecosistemas y Conservación en Bosques Amazónicos, Universidad de la Amazonía. osveti21@hotmail.com.

3 Docente Asistente, I. Agroecólogo. Ph.D. Grupo de Investigación en Agroecosistemas y Conservación en Bosques Amazónicos, Universidad de la Amazonía. juansuarez1@gmail.com

Fecha recepción: Marzo 13 de 2015

Fecha de aceptación: Mayo 11 de 2015

\section{RESUMEN}

El estudio se realizó en el departamento del Caquetá, municipio de Cartagena del Chairá con el objetivo de evaluar la contribución de los sistemas productivos en la generación de ingresos en familias cacaoteras. Se definieron 22 variables cualitativas y cuantitativas, que se agruparon en cinco categorías: (i) caracterización social, (ii) características de la finca, (iii) tamaño de la finca y uso del suelo, (iv) producción pecuaria, (v) producción agrícola y forestal. En torno a las cinco categorías se generaron grupos a partir de un Análisis de Correspondencia Múltiple (ACM). Finalmente, los grupos se conglomeraron en tres clúster que presentaron diferencias significativas $(\mathrm{P}<0,05)$ en las variables uso del suelo e ingresos por actividades agropecuarias. Las Fincas ganaderas grandes presentaron áreas en pasturas de 109,75 ha e ingresos anuales de $\$ 15.321 .250$, provenientes de la actividad pecuaria. Las fincas agrícolas pequeñas tuvieron áreas en bosque de 48,78 ha, y se obtuvieron ingresos anuales de $\$ 1.170 .000$ derivados del bosque. Las Fincas agropecuarias medianas presentaron áreas en cultivos agrícolas de 6,89 ha, sin embargo 
estas fincas obtuvieron ingresos no relacionados con la producción agropecuaria de $\$ 2.052 .916$. Se concluye que las familias cacaoteras requieren de diferentes actividades productivas para obtener ingresos debido a que el cultivo de cacao es de subsistencia.

Palabras clave: ACM, Actividades productivas, Clúster, Fincas.

\begin{abstract}
The study was conducted in the department of Caqueta, in the municipality of Cartagena del Chaira, with the objective of evaluating the contribution of production systems to the income generation of cocoa farming families. Twenty two qualitative and quantitative variables were defined, which were grouped into five categories: (i) social characterization, (ii) farm characteristics, (iii) farm size and soil use, (iv) livestock production, (v) agricultural and forestry production. Based on the five categories, groups were generated from a Multiple Correspondence Analysis (MCA). Lastly, the groups formed three clusters that showed significant differences $(\mathrm{P}<0.05)$ between soil use and income generated from agropecuary activities. The large livestock farms showed pasture areas of 109.75 ha and annual incomes of $\$ 15.321 .250$ COP, originating from livestock activity. The small agricultural farms had forest areas of 48.78 ha and annual incomes of $\$ 1.170 .000$ COP derived from the forest. The medium agropecuary farms had agricultural crops with an area of 6.89 ha; however, these farms showed incomes of $\$ 2.052 .916$ COP that were unrelated to the agropecuary production. It can be concluded that the cocoa farming families require different income-generating production activities, given that the cocoa crop is a subsistence activity.
\end{abstract}

Keywords: MCA, productive activities, cluster, farms.

\section{INTRODUCCIÓN}

Un sistema productivo se define como el conjunto de procesos de trabajo de cada sector de la producción, que comparten el mismo grado de desarrollo de las fuerzas productivas y que pueden identificarse por las propiedades de sus elementos participantes, la dinámica de las relaciones (organización del trabajo, estrategia adaptativa o de control) y la intensidad de uso del suelo (Parra, 1991).

A su vez, los sistemas productivos no están formados por explotaciones homogéneas. Existe diversidad con respecto a diferentes caracteres físicos, socioeconómicos y/o técnicos (Coronel de Renolfi y Ortuño, 2005). De acuerdo con Dufumier (1990) se destacan tres tipos de explotaciones: i) explotaciones familiares mercantiles, en las cuales los sistemas de producción utilizados aseguran el pleno empleo de la fuerza de trabajo familiar y garantizan un ingreso suficiente que posibilita la sobrevivencia de la familia, sin recurrir a la venta al exterior de la fuerza de trabajo; ii) explotaciones subfamiliares en las que los sistemas de producción no permiten asegurar un ingreso suficiente para la sobrevivencia de las familias y el pleno empleo de sus miembros, por consiguiente, la mano de obra familiar esta forzada a buscar trabajo fuera de la finca y las actividades productivas que se asumen tienden a satisfacer las necesidades de 
consumo de la familia a partir de una cantidad reducida de productos alimenticios de primera necesidad; iii) explotaciones multifamiliares en las que los sistemas de producción exigen una cantidad de fuerza de trabajo bastante superior a la que puede aportar una sola familia; por esto; es necesario recurrir al empleo de mano de obra exterior, la que puede ser pagada con dinero, en especie, por concesión de una parcela, $u$ otras formas.

Godar et al. (2012) explica que un pequeño agricultor que cultiva 100 ha de cacao bajo árboles de sombra tiene resultados económicos y ambientales diferentes del productor que desarrolla ganadería en la misma zona. De esta manera, la selección de los sistemas productivos depende de los intereses que desarrolla cada productor, según la escasez relativa de los recursos disponibles con el fin de maximizar los ingresos por hectárea (Dufumier, 1990), generando interacciones entre la agricultura de subsistencia, la producción de cultivos comerciales y otros medios de diversificación de los ingresos (Mertz et al. 2005).

Los sistemas diversificados de producción, tienen mayor estabilidad en los ingresos netos de las familias que dependen de ellos (Sáenz, 2012). En los sistemas productivos se promueve la diversificación de las fincas con arreglos agroforestales de cacao y puede dar como resultado: (i) reducción de la incertidumbre y el riesgo; (ii) alcance de servicios de mercado, (iii) mejoras en la situación financiera; (iv) aspiraciones y actividades del individuo; (v) mayores ingresos, y (vi) conexiones entre la familia (López et al. 2011).

El cultivo de cacao se desarrolla en gran medida bajo un sistema de producción campesina, es decir, el productor vive, trabaja y deriva la mayor parte de su sustento de la finca (SIC, 2012), debido a este característica socioeconómica demanda mano de obra aportada por familias, de manera que genera empleo directo e indirecto y es dinamizador de la economía en zonas que presentan problemáticas sociales (pobreza, desempleo, violencia y presencia de cultivos ilícitos) (Perdomo, 2012). El objetivo de esta investigación, fue evaluar la contribución de los sistemas productivos en la generación de ingresos para las familias cacaoteras del Bajo Caguán, Cartagena del Chaira.

\section{MATERIALES Y MÉTODOS}

Área de estudio. El presente estudio se desarrolló en el departamento del Caquetá, municipio de Cartagena del Chairá, zona del Bajo Caguán, que comprende 65 veredas distribuidas en 4 núcleos veredales (Santo Domingo, Remolinos del Caguán, Peñas Coloradas y Santa Fe) (Gobernación del Caquetá, 2012). Está zona se encuentra enmarcada dentro de las coordenadas $1^{\circ} 21^{\prime} 0^{\prime \prime}$ LN y $74^{\circ} 50^{\prime} 24^{\prime \prime}$ LO. Presenta una precipitación anual entre 2500 y 3500 mm, distribuidos en 10 meses, con una época relativamente seca entre diciembre y marzo; humedad relativa promedio del $90 \%$; temperatura media de $26^{\circ} \mathrm{C}$; evaporación de 970,5 mm.año ${ }^{-1}$; brillo solar de 1508 horas luz año ${ }^{-1}$; velocidad del viento promedio de 1,3 m.seg-1 y altura de $234 \mathrm{msnm}$ (Alcaldía de Cartagena del Chaira, 2012).

Recopilación de información. Se seleccionaron 50 fincas ubicadas en 27 veredas del Bajo Caguán, que presentaron áreas con arreglos agroforestales en diferentes etapas de crecimiento 
y/o producción. Para realizar la caracterización de fincas, se utilizó como base la metodología propuesta por Suárez (2009) a partir del diseño de encuestas tipo cuestionario semiestructurada. La aplicación de entrevistas se realizó por medio de reuniones con las familias cacaoteras, se recopilo información relacionada con: generalidades del productor y la finca, unidad familiar, técnica del manejo de la finca, economía y mercadeo de los productos agropecuarios y forestales, visión del productor y su familia.

Selección de variables. Se utilizaron 22 variables cualitativas y cuantitativas, agrupadas en cinco categorías: (i) caracterización social, (ii) características de la finca, (iii) tamaño de la finca y uso del suelo, (iv) producción pecuaria, (v) producción agrícola y forestal.

Variables de caracterización social. Se seleccionaron cuatro variables (Tabla 1 ).

- Nivel de educación de las familias: se clasificaron las familias en tres niveles de educa- ción: bajo (ninguna); medio (primaria) y alto (secundaria).

- Tamaño del hogar: se realizó el conteo del total de integrantes por familia y se establecieron tres categorías: bajo (menores a cuatro personas), medio (entre cuatro y seis personas) y alta (mayor a seis personas).

- Origen del jefe del hogar: se consideró el lugar de procedencia del jefe del hogar: misma comunidad, mismo departamento y otro departamento.

- Posición del hogar en el ciclo de vida: se promedió la edad de los integrantes de la familia y se definieron tres niveles: menor de 25 años, entre 25 y 40 años y mayor de 40 años.

Variables de caracterización de la finca. Se definieron cinco variables (Tabla 2).

- Forma de tenencia de la tierra: se clasificó en propietario, administrador y arrendatario.

Tabla 1. Variables y clases de caracterización social de familias con frecuencias observadas.

\begin{tabular}{l|l|l|l}
\hline Caracterización social & Clase & Código & $\mathbf{n}$ \\
\hline \multirow{3}{*}{ 1. Nivel de educación de las familias } & Ninguna & NE_B & 8 \\
& Primaria & NE_M & 38 \\
& Secundaria & NE_A & 4 \\
\hline \multirow{3}{*}{ 2. Tamaño del hogar (personas) } & $<4$ & NP_B & 35 \\
& $4-6$ & NP_M & 11 \\
& $>6$ & NP_A & 4 \\
\hline \multirow{3}{*}{ 3. Origen del jefe del hogar } & Misma comunidad & Mis_Com & 12 \\
& Mismo departamento & Mis_Dep & 2 \\
& Otro departamento & Otr_Dep & 36 \\
\hline \multirow{3}{*}{ 4. Posición del hogar en el ciclo de vida (edad) } & $<25$ & $<25$ años & 10 \\
& $25-40$ & $25-40$ años & 18 \\
& $>40$ & $>40$ años & 22 \\
\hline
\end{tabular}


- Años de ocupación: se estableció tres categorías de acuerdo con el número de años que tienen las familias ocupando la finca (menores de 10 años, entre 10 y 20 años y mayores de 20 años).

- Caminos de acceso: se distinguió las fincas que se encontraban ubicadas a orilla del río y camino de herradura.

- Principal actividad de la finca: se identificaron cuatro clases en torno a la principal actividad que se desarrolla en la finca (agrícola, pecuaria, forestal y ninguna).

- Proyección de la unidad productiva: se presentó lo que cada familia planea realizar en el futuro en su unidad productiva.
Tamaño de la finca y variables de usos del suelo. Se definieron cinco variables en torno a la distribución del uso del suelo en la finca (Tabla 3).

- Tamaño del predio: se clasificaron en: fincas pequeñas con un área menor a 152 ha, fincas medianas entre 152 y 300 ha, fincas grandes con un área mayor a 300 ha.

- Usos del suelo: se categorizó en tres niveles de acuerdo con el porcentaje de ocupación de cada uso en relación al área total del predio.

- Bosque y pasturas: bajo (menor al 30\%), medio (entre 30 y $60 \%$ ), alto (superior al 60\%).

- Regeneración natural, cultivos agrícolas permanentes y anuales: bajo (menor al $10 \%)$, medio (entre 10 y $35 \%$ ), alto (superior el 35\%).

Tabla 2. Variables y clases de características de la finca con frecuencias observadas.

\begin{tabular}{l|l|l|l}
\hline \multicolumn{1}{c|}{ Características de la finca } & \multicolumn{1}{c|}{ Clase } & \multicolumn{1}{c}{ Código } & \multicolumn{1}{c}{ n } \\
\hline \multirow{3}{*}{ 1. Forma de tenencia de la tierra } & Propietario & Prop & 48 \\
& Administrador & Adm & 1 \\
& Arrendatario & Arr_ & 1 \\
\hline \multirow{3}{*}{ 2. Años de ocupación } & $<10$ & $<10$ años & 27 \\
& $10-20$ & $10-20$ años & 18 \\
& $>20$ & $>20$ años & 5 \\
\hline \multirow{2}{*}{ 3. Caminos de acceso } & Orilla del río & Or_Rio & 21 \\
& Camino de herradura & Cam_He & 29 \\
\hline \multirow{5}{*}{ 4. Principal actividad de la finca } & Agrícola & Agric & 19 \\
& Pecuaria & Pec & 29 \\
& Forestal & Fores & 1 \\
& Ninguna & Ning & 1 \\
\hline \multirow{3}{*}{ 5. Proyección de la unidad productiva } & Cacao & Fort_Cac & 12 \\
& Ganadería & Fort_Ganad & 13 \\
& Cacao y ganado & Fort_CayGan & 15 \\
& Agrícola & Aum_Agri & 10 \\
\hline
\end{tabular}


Tabla 3. Variables y clases para tamaño de la finca y usos del suelo con frecuencias observadas.

\begin{tabular}{l|l|l|c}
\hline Tamaño de la finca y uso del suelo & Clase & Código & n \\
\hline \multirow{4}{*}{ 1. Tamaño del predio } & $<152$ ha & APred_B & 37 \\
& $152-300$ ha & APred_M & 9 \\
& $>300$ ha & APred_A & 4 \\
\hline \multirow{2}{*}{ 2. Bosque } & $<30 \%$ & ABosq_B & 11 \\
& $30-60 \%$ & ABosq_M & 16 \\
3. Pasturas & $>60 \%$ & ABosq_A & 23 \\
\hline \multirow{5}{*}{ 4. Regeneración natural } & $<30 \%$ & APast_B & 30 \\
& $30-60 \%$ & APast_M & 16 \\
& $>60 \%$ & APast_A & 4 \\
\hline \multirow{2}{*}{ 5. Cultivos agrícolas permanentes y anuales } & $<10 \%$ & AReg_B & 41 \\
& $10-35 \%$ & AReg_M & 6 \\
& $>35 \%$ & AReg_A & 3 \\
\hline
\end{tabular}

Variables de producción. Las variables de producción describen los sistemas productivos de las familias. Se consideraron tres sistemas de producción: pecuario, agrícola y forestal. Dentro de la producción pecuaria se seleccionaron cinco variables en torno a: número de vacunos, aves de corral, porcinos, tipo de ganadería bovina y producción de leche. Para la producción agrícola y forestal se establecieron tres variables: área sembrada en cacao, producción de cacao y explotación de especies maderables para comercialización (Tabla 4).

Análisis de datos. Las variables cuantitativas fueron categorizadas en descriptores cualitativas con tablas de contingencia, como sugieren Solano et al. (2000). Se realizó un Análisis de Correspondencia Múltiple $(\mathrm{ACM})$ entre categorías de variables, identificando grupos con su respectivo número de fincas. Al relacionar la anterior información se obtuvieron clúster de fincas, en los que se empleó el Análisis de Varianza (ANDEVA) con el software estadístico InfoStat versión 2014 (Di Rienzo et al., 2014). Para determinar diferencias estadísticas entre variables de uso del suelo e ingresos se utilizó la prueba de comparación de medias de LSD Fisher $(\mathrm{p}<0,05)$.

\section{RESULTADOS Y DISCUSIÓN}

Relación entre variables de caracterización social de las familias. En el conjunto de las variables de caracterización social se generó una tipificación de tres grupos de fincas, que comparten características similares: (i) familias con un nivel educativo alto, procedentes de la misma comunidad, tamaño del hogar medio y ciclo de vida bajo ( $F A M, \mathrm{n}=12$ ); (ii) familias con nivel educativo medio, procedentes del mismo departamento, ciclo de vida medio y tamaño del hogar alto $(F M B, \mathrm{n}=24)$ y (iii) familias con un nivel educati- 
vo bajo, procedentes de otro departamento, tamaño del hogar bajo y ciclo de vida alto (FBA, $\mathrm{n}=14$ ) (Figura 1). Como han señalado Orozco et al. (2008) en Talamanca-Costa Rica, las familias están conformadas por un promedio de cuatro miembros y el $40 \%$ de los miembros de la familia, incluyendo el propietario, ha completado la educación primaria, características similares a lo obtenido en la tipificación $F M B$. Por otra parte, Vigneri (2007) expresa que una familia cacaotera está dirigida por hombres en sus 40 años de edad, con una edad promedio de 49 años, lo anterior explica el ciclo de vida alto encontrado en la tipificación FBA.

Tabla 4. Variables y clases para sistemas de producción pecuario, agrícola y forestal con frecuencias observadas.

\begin{tabular}{|c|c|c|c|}
\hline Variable & Clase & Código & n \\
\hline \multicolumn{4}{|c|}{ Pecuario } \\
\hline 1. Tipo de ganadería bovina & $\begin{array}{l}\text { Ninguna } \\
\text { Carne } \\
\text { Leche } \\
\text { Doble propósito }\end{array}$ & $\begin{array}{l}\text { Gan_Ning } \\
\text { Gan_Car } \\
\text { Gan_Lec } \\
\text { Gan_Dprop }\end{array}$ & $\begin{array}{l}14 \\
13 \\
8 \\
15\end{array}$ \\
\hline 2. Número de vacunos & $\begin{array}{l}0 \\
<20 \\
20-60 \\
>60\end{array}$ & $\begin{array}{l}\text { Vac_N } \\
\text { Vac_B } \\
\text { Vac_M } \\
\text { Vac_A }\end{array}$ & $\begin{array}{l}14 \\
13 \\
12 \\
11\end{array}$ \\
\hline 3. Número de aves de corral & \begin{tabular}{|l}
0 \\
$<20$ \\
$20-50$ \\
$>50$ \\
\end{tabular} & $\begin{array}{l}\text { Ave_N } \\
\text { Ave_B } \\
\text { Ave_M } \\
\text { Ave_A }\end{array}$ & $\begin{array}{l}21 \\
16 \\
11 \\
2 \\
\end{array}$ \\
\hline 4. Número de porcinos & \begin{tabular}{|l}
0 \\
$<10$ \\
$10-20$ \\
$>20$ \\
\end{tabular} & $\begin{array}{l}\text { Por_N } \\
\text { Por_B } \\
\text { Por_M } \\
\text { Por_A }\end{array}$ & $\begin{array}{l}24 \\
16 \\
7 \\
3 \\
\end{array}$ \\
\hline 5. Producción de leche (litros año ${ }^{-1}$ ) & \begin{tabular}{|l}
0 \\
$<3000$ \\
$3000-10000$ \\
$>10000$ \\
\end{tabular} & $\begin{array}{l}\text { LtLec_N } \\
\text { LtLec_B } \\
\text { LtLec_M } \\
\text { LtLec_A }\end{array}$ & $\begin{array}{l}8 \\
6 \\
6 \\
30 \\
\end{array}$ \\
\hline \multicolumn{4}{|c|}{ Agrícola y forestal } \\
\hline 1. Área sembrada en cultivo de cacao (ha) & \begin{tabular}{|l|}
0 \\
$<4$ \\
$4-8$ \\
$>8$ \\
\end{tabular} & $\begin{array}{l}\text { Acac_N } \\
\text { Acac_B } \\
\text { Acac_M } \\
\text { Acac_A }\end{array}$ & $\begin{array}{l}2 \\
38 \\
7 \\
3 \\
\end{array}$ \\
\hline 2. Producción de cacao (kilo año-1) & $\begin{array}{l}\text { Ninguno } \\
0 \\
<450 \\
450-1350 \\
>1350\end{array}$ & $\begin{array}{l}\text { KgCac_N } \\
\text { KgCac_0 } \\
\text { KgCac_B } \\
\text { KgCac_M } \\
\text { KgCac_A }\end{array}$ & $\begin{array}{l}2 \\
19 \\
20 \\
7 \\
2\end{array}$ \\
\hline 3. Explotación de madera (bloques año $0^{-1}$ ) & $\begin{array}{l}\text { Ninguno } \\
<150 \\
>150\end{array}$ & $\begin{array}{l}\text { BMad_N } \\
\text { BMad_B } \\
\text { BMad_A }\end{array}$ & $\begin{array}{l}44 \\
3 \\
3\end{array}$ \\
\hline
\end{tabular}




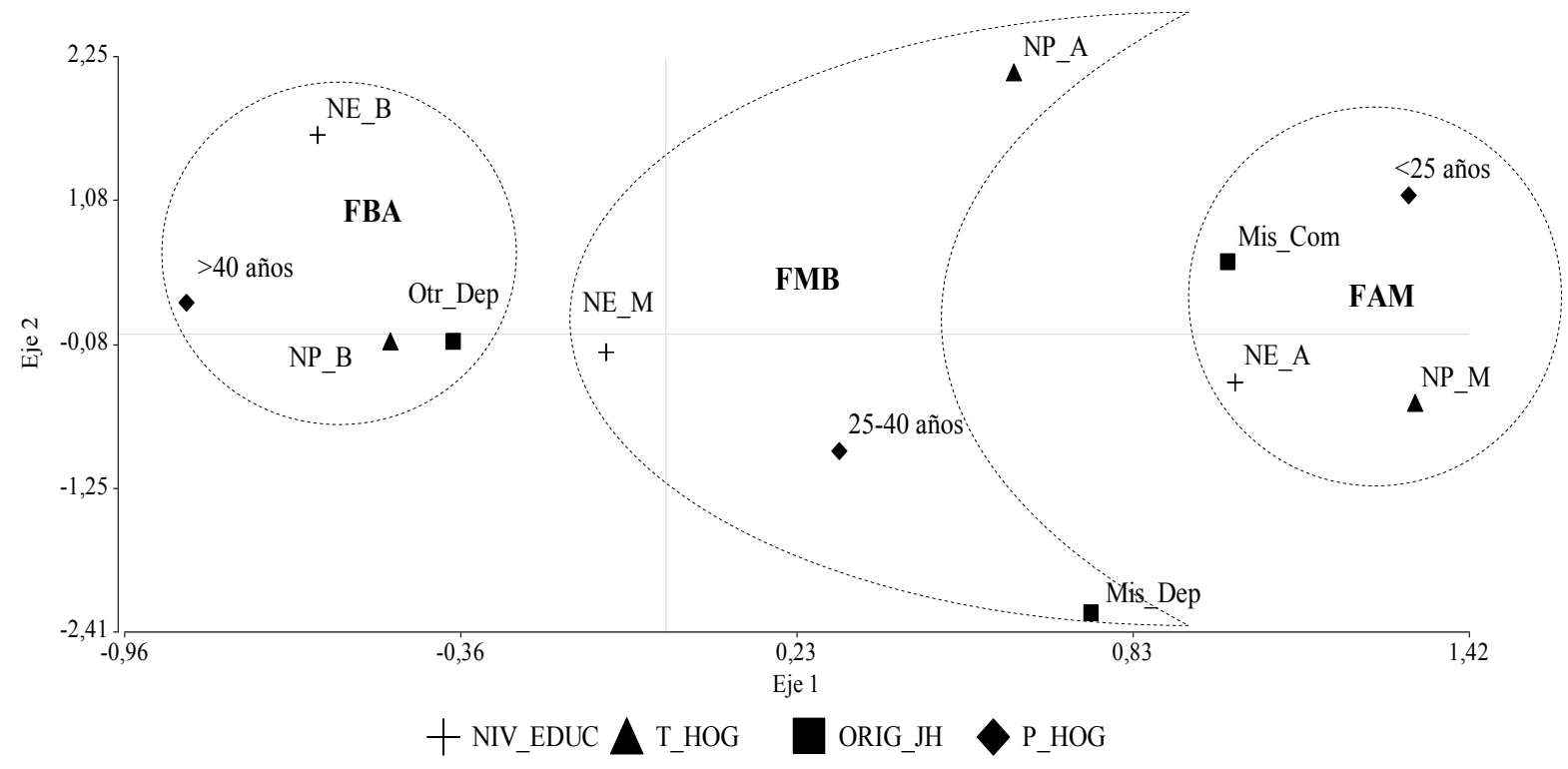

Figura 1. Relación entre las características sociales de las familias cacaoteras.

Relación entre variables de características de la finca. En la Figura 2, se muestra la relación entre la forma de tenencia de la tierra, años de ocupación del predio, caminos de acceso, principal actividad desarrollada y proyección productiva. Se identificaron dos grupos de fincas: (i) FPP: fincas de propietarios con enfoque pecuario, asentadas entre 10 y más de 20 años, con caminos de acceso de herradura, proyección ganadera y cacaotera $(n=32)$. (ii) FPA: fincas de propietarios con enfoque agrícola, asentadas hace menos de 10 años, ubicadas a orilla del río y con proyección cacaotera $(\mathrm{n}=18)$. Godar et al. (2012) exponen, que en relación con el acceso a los centros urbanos, los pequeños agricultores están cerca de la carretera en comparación con productores ganaderos, tal como sucede en el grupo FPA. De acuerdo con Orozco et al. (2008), los productores residen y trabajan sus tierras desde hace 16 años en promedio, aunque hay productores que trabajan sus tierras desde hace
70 años y otros que han llegado a la zona desde hace apenas un año, lo anterior explica la forma de asentamiento de las familias cacaoteras en los grupos de fincas FPP y FPA.

\section{Relación entre tamaño de la finca y uso del suelo.} En cuanto al tamaño de la finca y las variables de uso del suelo se encontró tres grupos de predios: (i) fincas pequeñas con un área en regeneración natural y pasturas alta, área en bosque baja y área en cultivos anuales y permanentes media, lo que significa que son fincas con múltiples usos del suelo (FEM, n=22); (ii) fincas medianas con un área en bosque y pasturas media, áreas bajas en regeneración natural y cultivos anuales y permanentes, es decir, fincas con enfoque agropecuario (FEAG, $\mathrm{n}=24$ ) y (iii) fincas grandes con área en bosque alta, regeneración natural, pasturas, cultivos anuales y permanentes bajo, que se definen como fincas con enfoque agrícola $($ FEA, n=4) (Figura 3). 


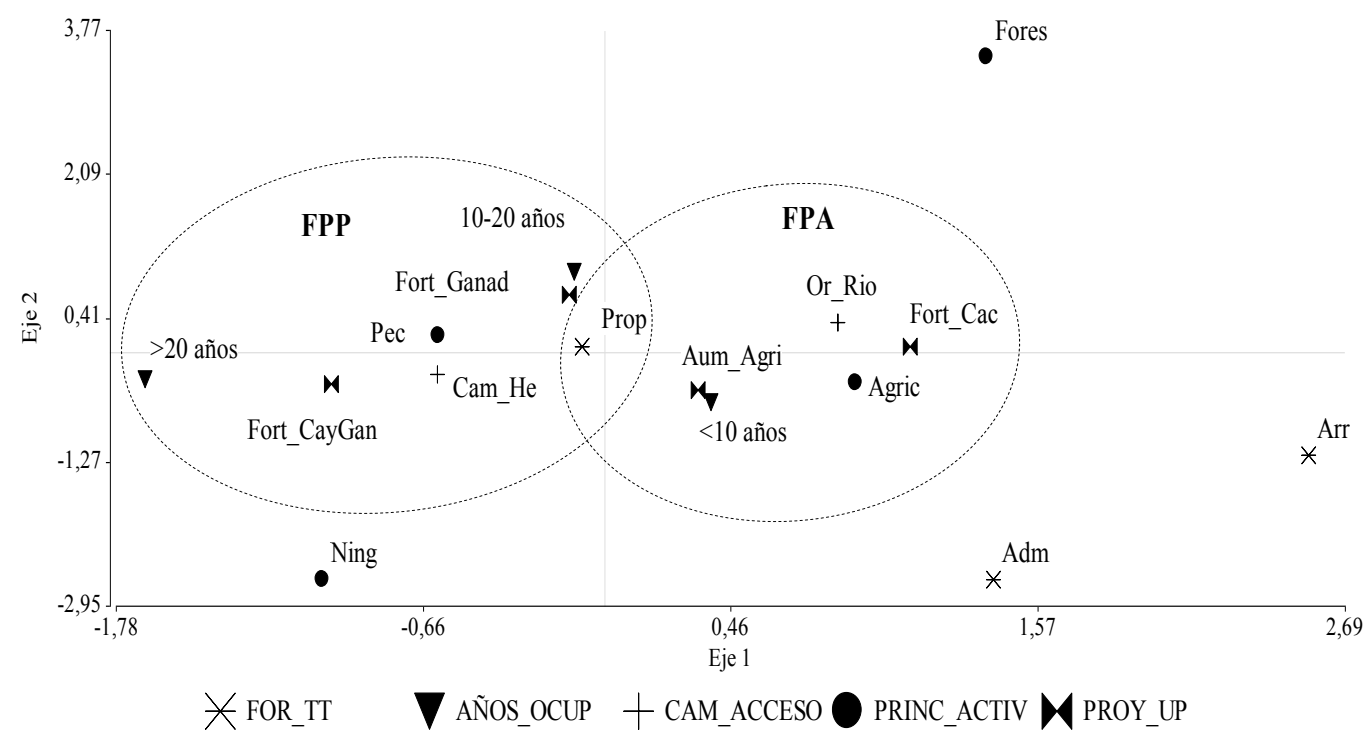

Figura 2. Relación entre variables de características de la finca.

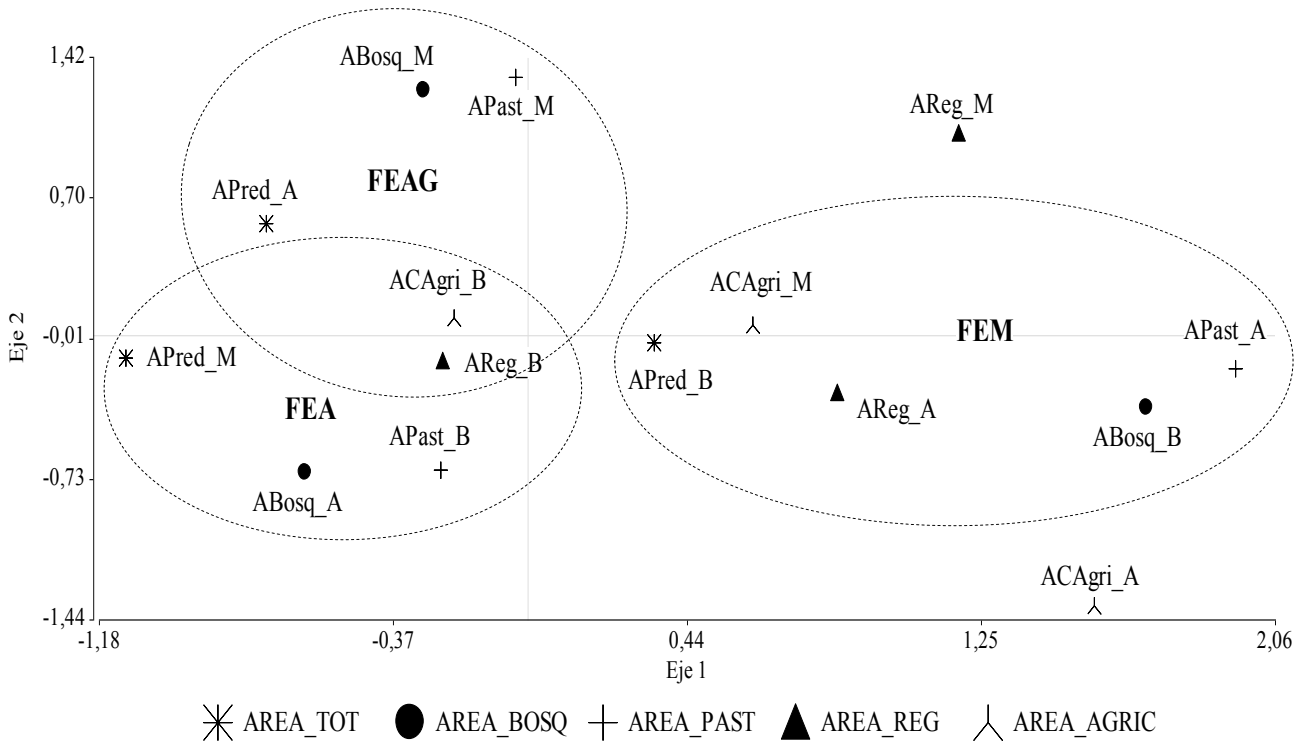

Figura 3. Relación entre tamaño de la finca y uso del suelo.

En los tres grupos anteriormente descritos, se destacan como principales usos del suelo las áreas en bosque y pasturas, a diferencia de lo expuesto por Somarriba et al. (2003) los principales usos de la tierra son: cacao, cacao con banano, banano, bosque, huertos caseros y potreros.
Los grupos FEAG y FEA presentan la menor área en cultivos anuales y permanentes, que de acuerdo con Preciado et al. (2011), las unidades productivas tienen de 1 a 5 ha dedicas a la agricultura. Paredes (2001) explica que el área destinada para los cultivos agrícolas no 
asciende a más de 5 ha y no son incorporadas a la actividad productiva porque se tienen tierras muy alejadas, que originan altos costos de producción. Schroth y Ruf (2014) señalan que las fincas grandes son más diversificadas que las pequeñas explotaciones, lo anterior difiere a lo encontrado en el presente estudio, ya que la diversificación en los usos del suelo es mayor en FEM, lo cual se puede ver reflejado en fuentes alternativas de ingresos (Gyau et al. 2014).

\section{Relación entre variables del sistema productivo} pecuario. Para las variables del sistema productivo pecuario se identificaron cuatro grupos de fincas, que muestran un fuerte indicio de que las familias en estudio se dedican a la ganadería y/o cría de especies menores (Toruño, 2012): (i) fincas porcícolas y avícolas (FAP, $\mathrm{n}=3)$, poseen un alto número de cerdos y aves de corral pero carecen de producción bovina; (ii) fincas con ganadería tipo carne, con menos de 20 cabezas de ganado, una producción de leche entre 0 y 3000 litros año-1 ${ }^{-1}$ con ausencia de cerdos $\mathrm{y}$ aves de corral dentro de su sistema pecuario
$(F C B, \mathrm{n}=8)$; (iii) fincas con ganadería tipo leche, con una producción entre 3000 y 10000 litros. año ${ }^{-1}$, un número de vacunos entre 20 y 60, además de poseer menos de 20 aves de corral y porcinos (FLM, $\mathrm{n}=10$ ); (iv) fincas con ganadería doble propósito, con más de 60 cabezas de ganado y una producción alta de leche $(>10000$ litros año-1 ${ }^{-1}$, con un número de porcinos y aves de corral medio (FDA, n=29) (Figura 4).

El financiamiento de la inversión en el sector ganadero requiere de excedentes considerables de otras actividades o de acceso al crédito (Abdulai y Crole-Rees, 2001); en la zona del Bajo Caguán, la disponibilidad de crédito es alta, lo cual contribuye a una alta participación de los hogares en la actividad ganadera. Mora (2012) desde el punto de vista administrativo, explica que los sistemas ganaderos con la producción de leche manejan un flujo de caja constante, el levante y la ceba se reservan como ahorro o respaldo en emergencias económicas. Lo anterior se presenta en el sistema productivo

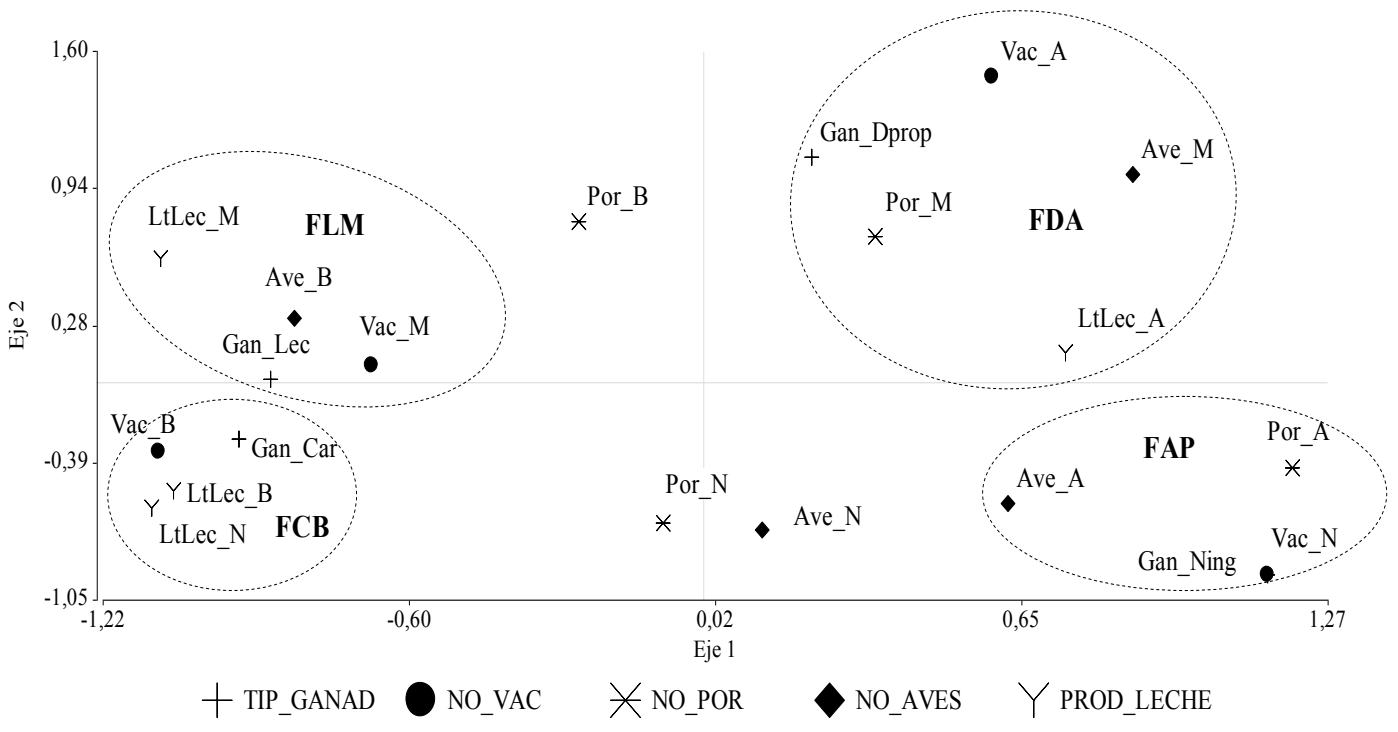

Figura 4. Relación entre variables del sistema productivo pecuario. 
ganadero que posee el grupo FDA. Sin embargo, en el grupo FAP predominan aves de corral y cerdos. De acuerdo con Porro et al. (2014), la crianza de estas especies, es pertinente para complementar los ingresos pecuarios.

\section{Relación entre variables del sistema productivo} agrícola y forestal. Se identificaron dos grupos de fincas entre las variables de producción agrícola y forestal: (i) fincas con más de 8 hectáreas en cacao, producción superior a $450 \mathrm{~kg}$ de cacao año ${ }^{-1}$, explotación de madera de 150 bloques año $0^{-1}(F C M A, \mathrm{n}=4)$; (ii) fincas con menos de 8 hectáreas en cacao, producción de cacao inferior a $450 \mathrm{~kg}$ de cacao año $0^{-1}$ y ninguna explotación de madera $(F C M B, \mathrm{n}=46)$ (Figura 5).

El grupo FCMB cuenta con la superficie óptima para mantener la unidad familiar, de acuerdo con lo señalado por el IICA (2001) corresponde a un área promedio de 3,3 hectáreas de cacao; en consecuencia, Asare et al. (2014) exponen que el cacao es la actividad de cultivo dominante en el área de estudio con tamaños medios de 2,45 ha para el cacao; de igual forma, se debe tener en cuenta que la mayoría de los agricultores cultivan el cacao en una sola área, mientras que otros agricultores prefieren tener áreas de cacao separadas (Dumont et al., 2014).

Con respecto a la producción de cacao en el grupo FCMA, Vaast y Somarriba (2014) constatan que las plantaciones de cacao en África y las Américas cubren áreas entre 0,25 y $5 \mathrm{ha}$, con rendimientos de $300 \mathrm{a} 600 \mathrm{~kg} \mathrm{ha}^{-1}$ año $0^{-1}$ de cacao. Lo anterior muestra que los beneficios derivados de áreas superiores a 8 ha con rendimientos de $450 \mathrm{~kg}^{2}$ año ${ }^{-1}$ en la zona del Bajo Caguán, no son equivalentes a los altos rendimientos en áreas más pequeñas (Cerda et al., 2014; Deheuvels et al., 2014).

Relación entre categorías estudiadas. Se establecieron tres clúster de fincas a partir de la relación entre las cinco categorías previamente estudiadas (Figura 6), que presentan las siguientes características:

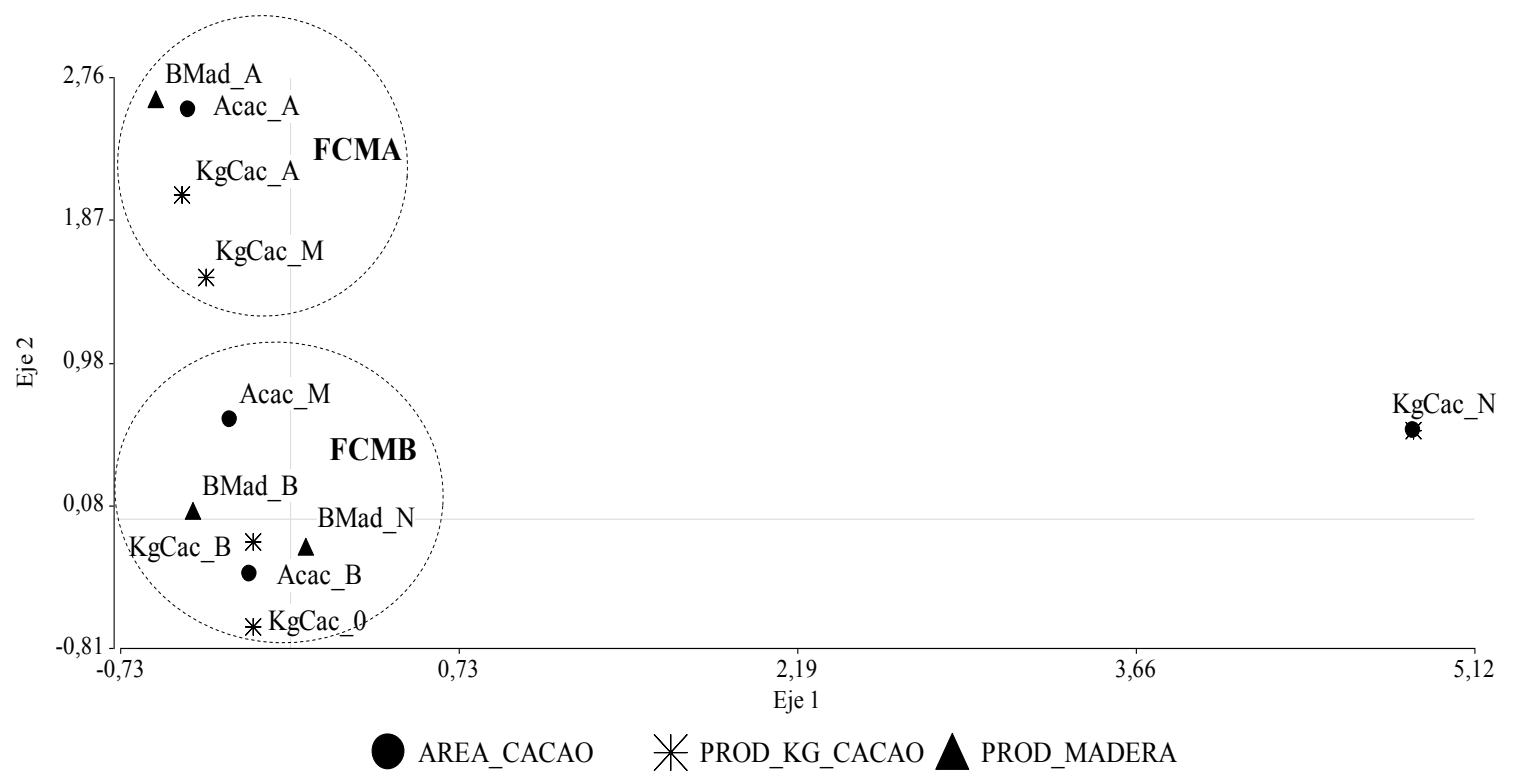

Figura 5. Relación entre variables del sistema productivo agrícola y forestal. 


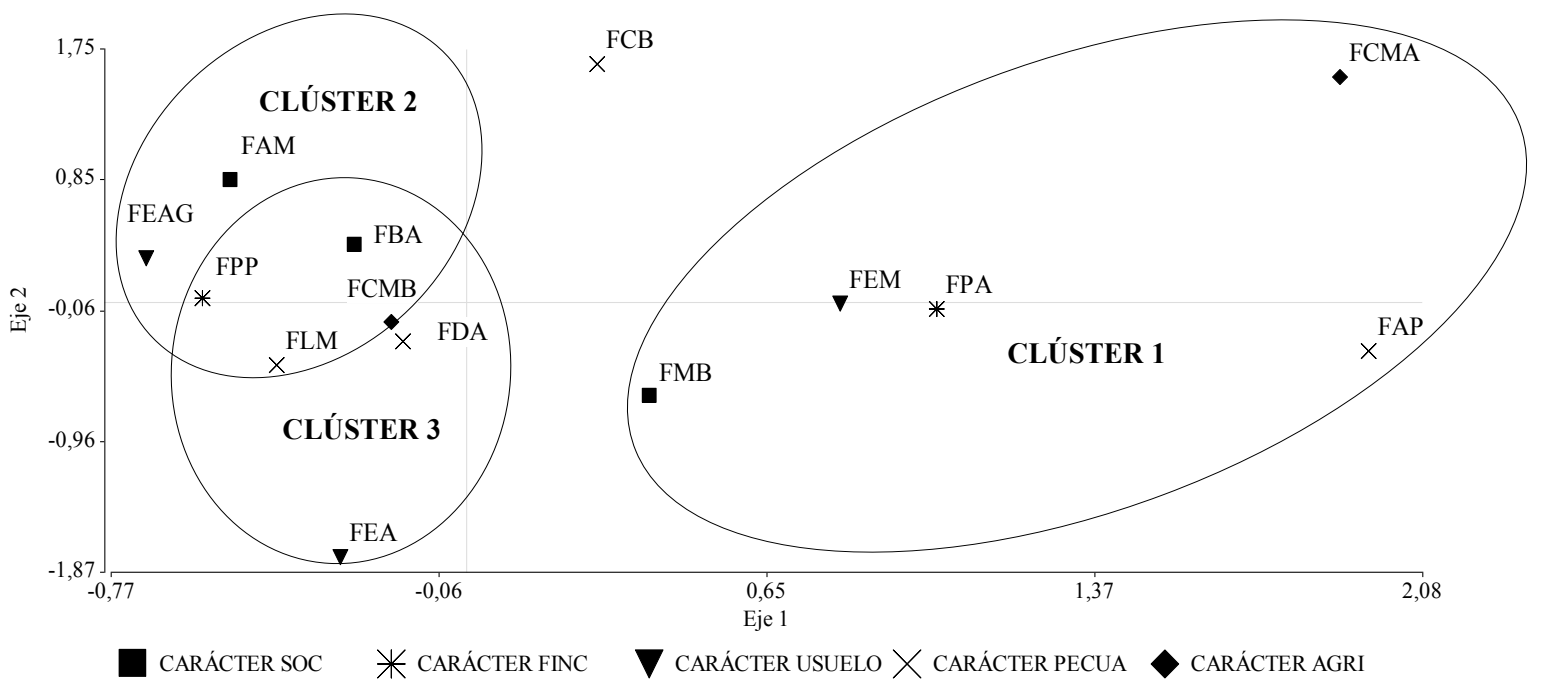

Figura 6. Relación entre variables de cinco categorías estudiadas: características sociales, de la finca, uso del suelo, sistema productivo pecuario, agrícola y forestal.

Clúster 1: Fincas agrícolas pequeñas: refleja el grado de dependencia de las fincas con múltiples usos del suelo (FEM), basada en la cría de cerdos y aves (FAP), alta producción de cacao y explotación de madera (FCMA), al estar ubicadas a orilla del río (FPA) con familias procedentes del mismo departamento $(F M B)(\mathrm{n}=16)$.

Clúster 2: Fincas agropecuarias medianas: las fincas con enfoque agropecuario (FEAG) se relacionan directamente con la producción de ganadería tipo leche (FLM), pero presentan una correlación negativa con la producción de cacao y madera $(F C M B)$, porque se dificulta la comercialización por caminos de herradura $(F P P)$, son familias procedentes de la misma comunidad (FAM) o de otro departamento $(F B A)(\mathrm{n}=30)$.

Clúster 3: Fincas ganaderas grandes: las fincas con enfoque agrícola (FEA) presentan la dicotomía entre estrategias productivas que incluyen cultivos y bosque (FCMB), con ganadería tipo leche (FLM) o doble propósito (FDA). Los caminos de herradura $(F P P)$ no influyen en la comercialización de productos y corresponde a familias procedentes de otro departamento $(F B A)(n=4)$.

Dumont et al. (2014) exponen que los agricultores locales tienen parcelas más pequeñas que los agricultores migrantes, que generalmente adquieren grandes áreas para hacer la migración de mérito, situación que se presenta en las Fincas ganaderas grandes porque las familias proceden de otros departamentos. Así mismo, este clúster presenta sistemas de producción homogéneos orientados exclusivamente a la ganadería; sin embargo, se siembran cultivos anuales para consumo interno (Godar et al., 2012).

Ofori y Asafu (2011) han demostrado que los agricultores con niveles relativamente altos de educación tienen mayores niveles de ineficiencia técnica, dado que tienden a tener otras fuentes de ingresos, a los que dedican más atención a expensas de la gestión de sus explotaciones, situación que se evidencia en las Fincas agropecuarias medianas. 
Perz (2001) señala que los hogares con mayor número de integrantes, suelen plantar cultivos perennes como: cacao, café, coco, entre otros; por ello, la disponibilidad de mano de obra familiar es crucial para tener las plantas perennes productivas. Lo anterior es coincidente con las particularidades de las Fincas agrícolas pequeñas, porque se presenta un tamaño del hogar alto, que facilita la vinculación de los miembros de la familia a las diferentes actividades de la finca.

\section{Fuentes de ingresos de familias cacaoteras.}

Se presentaron diferencias significativas entre grupos de fincas en superficie y uso del suelo para las variables: área total, bosque, regeneración natural y pasturas, en consecuencia, los tres grupos de fincas reflejan las mayores áreas destinadas a bosque seguido de pasturas (Tabla. 5). Lo anterior se debe a la conversión de bosques en áreas de cultivo y campos de pastoreo (Sánchez y Aide, 2013), a través de los sistemas de roza y quema que son los pilares de la producción agrícola en estas áreas como afirman Mertz et al. (2005).

En cuanto a la dependencia de las fincas a tres tipos de ingresos (derivados del bosque, producción agropecuaria y no relacionados con producción agropecuaria), se encontró que todos los clúster son altamente dependientes de la producción agropecuaria (>70\% del ingreso total) (Tabla 5). En contraste, los resultados de la Prueba de Comparación de Fisher indican que los ingresos derivados del bosque y no relacionados con la producción agropecuaria no son estadísticamente diferentes. Sin embargo, se establece que los usos del suelo se encuentran directamente relacionados con la diversificación de las fuentes de ingresos de los hogares (Caviglia y Sills, 2005).
Como se señaló, el ingreso por producción agropecuaria domina en los tres clúster, lo agrícola y lo pecuario son factores diferenciadores importantes entre fincas. Los mayores ingresos se obtuvieron en Fincas ganaderas grandes (88,75\% del ingreso total), alrededor del $48 \%$ del ingreso total de la unidad de producción familiar proviene de la ganadería (Nahed et al., 2013) y el $40 \%$ proviene de cultivos tradicionales exportables como cacao y alimentos básicos o de subsistencia (maíz, arroz, yuca, plátano, entre otros) (Quaye et al., 2010). En Fincas agrícolas pequeñas, se prefiere la inversión en cultivos perennes y la formación de los pastos como el indicador de activos de capital (Porro et al., 2015) ya que la producción agrícola representa el $48 \%$ del ingreso total, lo que indica que el cacao y cultivos asociados (perennes y/o anuales) son la principal fuente de ingresos para las comunidades pobres (Deheuvels et al., 2012).

Los ingresos derivados del bosque son importantes para todos los clústeres debido a que la leña es el producto forestal más omnipresente (Porro et al., 2014); aunque, a causa del aserrío de madera, las Fincas agrícolas pequeñas presentan el $18,56 \%$ del ingreso total. La dependencia de los bosques para estos hogares está definida por el capital físico, como las sierras de cadena y el capital social que facilita el acceso al bosque (Ruf, 2011 y Uberhuaga et al., 2012). Además, a medida que la mano de obra es familiar, el bosque se corta continuamente en pequeñas porciones, se utiliza para la madera, frutas y extracción de semillas, así como para las actividades extractivas tradicionales (Costa et al., 2012).

El trabajo asalariado, es una de las principales fuentes de ingresos en los hogares (Somarriba et 


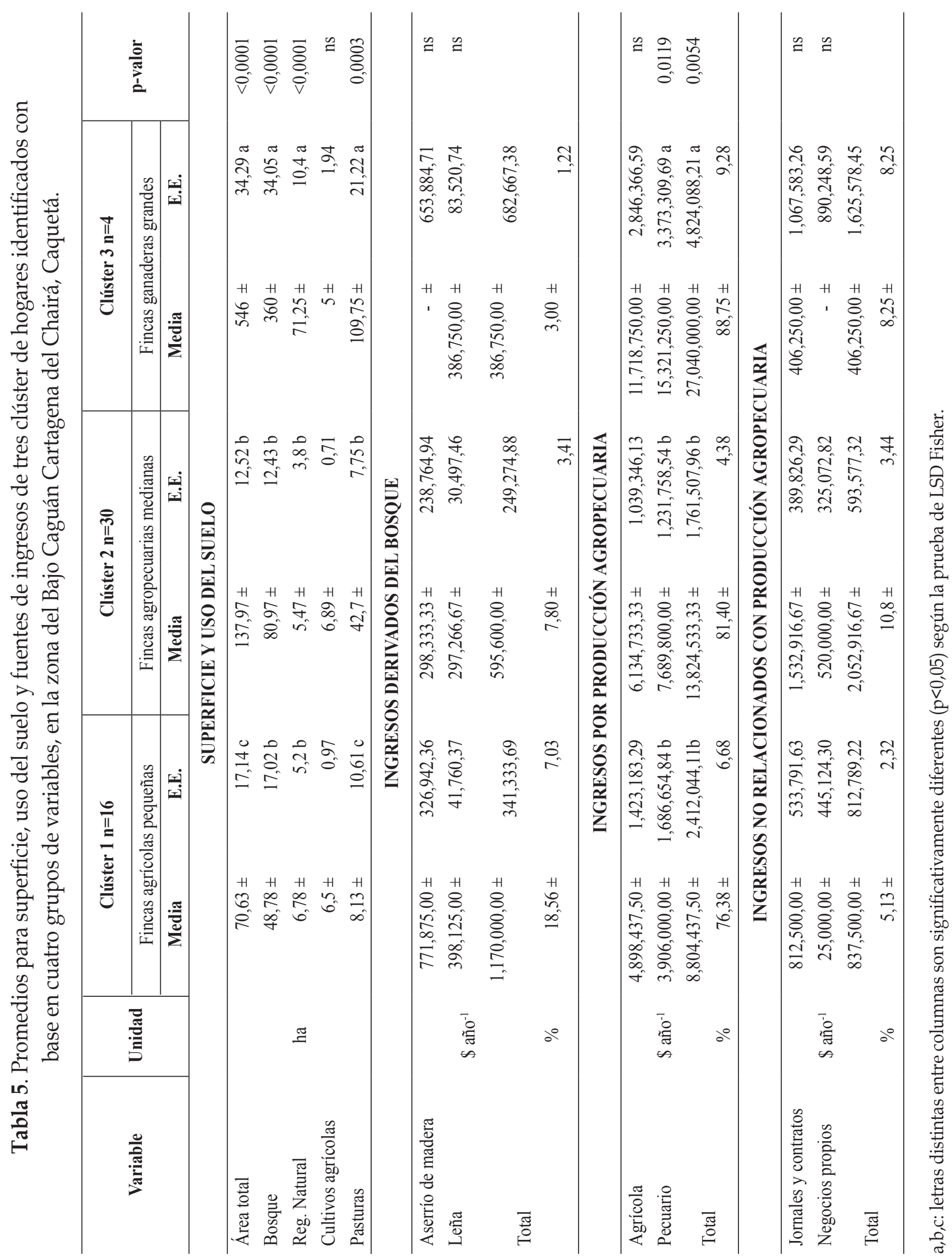


al. 2014). En consecuencia, las Fincas agropecuarias medianas presentaron los mayores ingresos provenientes de actividades no agropecuarias $(10,8 \%)$. Este resultado es consistente con los datos reportados por Abdulai y Crole-Rees (2001), que muestran que las actividades no agrícolas en efectivo proporcionan sólo el $6 \%$ de los ingresos totales de los hogares. Dado que los ingresos percibidos por las actividades no agrícolas es generalmente pequeño, pero constante, éste puede desempeñar un papel vital para la supervivencia de los hogares rurales pobres y generar la capacidad de invertir en la producción agrícola (Knudsen y Fold, 2011; Pédelahore, 2014).

\section{CONCLUSIONES}

Las Fincas agrícolas pequeñas obtienen menor ingresototalanual, tienenmenoresoportunidades de desarrollar producción bovina y trabajo no agropecuario.

Se presenta una correlación positiva entre el ingreso pecuario y el área en pasturas, las Fincas ganaderas grandes se especializan en la producción de bovinos de carne y/o leche.

Las actividades no agrícolas se consideran menos riesgosas que la producción de cultivos. Las familias estudiadas no presentaron dependencia de los ingresos no agropecuarios. Esta fuente de ingresos, se utiliza fuera de la temporada agrícola o cuando se requiere.

\section{AGRADECIMIENTOS}

A COLCIENCIAS mediante fondos BID en el marco del Convenio Especial de Cooperación 590/2012 para el desarrollo del proyecto de investigación "Caracterización y tipificación de plantaciones tradicionales de Theobroma cacao L. como estrategia de diseño e implementación de arreglos agroforestales en el Bajo Caguán (Cartagena del Chairá)", ejecutado por la Universidad de la Amazonía y el Comité de Cacaoteros de Remolinos del Caguán y SuncillaCHOCAGUAN.

\section{REFERENCIAS}

ABDULAI, A. y CROLE-REES, A. 2001. Determinants of income diversification amongst rural households in Southern Mali. Food policy. 26(4):437 - 452.

ALCALDÍA DE CARTAGENA DEL CHAIRA. 2012. Nuestro municipio: geografía. En: Sitio oficial de Cartagena del Chairá en Caquetá, Colombia. En: http://www.cartagenadelchaira-caqueta.gov.co/ informacion_general.shtml\#geografia. 1 p; consulta: octubre, 2014.

ASARE, R., AFARI-SEFA, V., OSEI-OWUSU, Y., y PABI, O. 2014. Cocoa agroforestry for increasing forest connectivity in a fragmented landscape in Ghana. Agroforestry Systems. 88(6):1143 - 1156.

CAVIGLIA-HARRIS, J. y SILLS, E. 2005. Land use and income diversification: comparing traditional and colonist populations in the Brazilian Amazon. Agricultural Economics. 32(3):221 - 237.

CERDA, R., DEHEUVELS, O., CALVACHE, D., NIEHAUS, L., SAENZ, Y., KENT, J., VILCHEZ, S., VILLOTA, A., MARTINEZ, C. y SOMARRIBA, E. 2014. Contribution of cocoa agroforestry systems to family income and domestic consumption: looking toward intensification. Agroforestry Systems. 88(6):957 - 81.

CORONEL DE RENOLFI, M. y ORTUÑO, S. 2005. Tipificación de los sistemas productivos agropecuarios en el área de riego de Santiago del Estero, Argentina. En: Portal de revistas científicas y arbitradas de la UNAM. En: http://www.ejournal.unam.mx/ pde/pde140/PDE14004.pdf. 26 p; consulta: febrero, 2015. 
COSTA, L., MIRANDA, I., GRIMALDI, M., SILVA, M., MITJA, D. y LIMA, T. 2012. Biomass in different types of land use in the Brazil's 'arc of deforestation'. Forest Ecology and Management. 278:101 - 109.

DEHEUVELS, O., AVELINO, J., SOMARRIBA, E. y MALEZIEUX, E. 2012. Vegetation structure and productivity in cocoa-based agroforestry systems in Talamanca, Costa Rica. Agriculture, Ecosystems \& Environment. 149:181 - 188.

DEHEUVELS, O., ROUSSEAU, G., QUIROGA, G., FRANCO, M., CERDA, R., MENDOZA, S. y SOMARRIBA, E. 2014. Biodiversity is affected by changes in management intensity of cocoa-based agroforests. Agroforestry Systems. 88(6):1081 1099.

DI RIENZO, J., CASANOVES, F., BALZARINI, M., GONZALEZ, L., TABLADA, M., y ROBLEDO, C. 2014. InfoStat versión 2014. Grupo InfoStat, FCA, Universidad Nacional de Córdoba, Argentina. En: http://www.infostat.com.ar; consulta: octubre, 2014.

DUFUMIER, M. 1990. Importancia de la tipología de unidades de producción agrícolas en el análisis de diagnóstico de realidades agrarias. pp. 63-81. En: Escobar, G. y Berdegué, J. (eds). Tipificación de sistemas de producción agrícola. Centro Latinoamericano para el Desarrollo Rural. Santiago de Chile, Chile. 284 p.

DUMONT, E., GNAHOUA, G., OHOUO, L., SINCLAIR, F. y VAAST, P. 2014. Farmers in Côte d'Ivoire value integrating tree diversity in cocoa for the provision of ecosystem services. Agroforestry Systems. 88(6):1047 - 1066.

GOBERNACIÓN DEL CAQUETÁ. 2012. Municipio Cartagena del Chairá: división política. En: Gobernación del Caquetá, República de Colombia. En: http:/ / www.caqueta.gov.co/index.php/nuestro-departamento/mapas/234-municipio-cartagena-del-chairadivision-politica. 1 p; consulta: octubre, 2014.
GODAR, J., TIZADO, E., POKORNY, B. y JOHNSON, J. 2012. Typology and characterization of amazon colonists: a case study along the Transamazon highway. Human Ecology. 40(2):251 - 267.

GYAU, A., SMOOT, K., KOUAME, C., DIBY, L., KAHIA, J. y OFORI, D. 2014. Farmer attitudes and intentions towards trees in cocoa (Theobroma cacao L.) farms in Côte d'Ivoire. Agroforestry Systems. 88(6):1035 - 1045.

INSTITUTO INTERAMERICANO DE COOPERACIÓN PARA LA AGRICULTURA (IICA). 2001. Acuerdo Sectorial de Competitividad de la Cadena del Cacao y su Agroindustria. En: IICA Colombia. En: http:/ / repiica.iica.int/DOCS/B0125E/B0125E. PDF. 40 p; consulta: Octubre, 2014.

KNUDSEN, M. y FOLD, N. 2011. Land distribution and acquisition practices in Ghana's cocoa frontier: the impact of a state-regulated marketing system. Land Use Policy. 28(2):378 - 387.

LÓPEZ, M., VILLANUEVA, C., HÄNSEL, G.y CHI, H. 2011. Evaluación económica y ambiental de fincas diversificadas con café y ganadería en la Zona Sur de Costa Rica. pp. 185-201. En: Villanueva, C.; Sepúlveda, C.; Ibrahim, M. (eds.). Manejo agroecológico como ruta para lograr la sostenibilidad de fincas con café y ganadería. Primera edición. CATIE. Turrialba, Costa Rica. 260 p.

MERTZ, O., WADLEY, R. y CHRISTENSEN, A. 2005. Local land use strategies in a globalizing world: Subsistence farming, cash crops and income diversification. Agricultural Systems. 85(3):209 - 215.

MORA, D. 2012. Sistema de producción a pequeña escala de dulce de leche caprino en Costa Rica. Agronomía Mesoamericana. 23(1):151 - 158.

NAHED-TORAL， J., VALDIVIESO-PÉREZ, A., AGUILAR-JIMÉNEZ, R., CÁMARA-CORDOVA, J. y GRANDE-CANO, D. 2013. Silvopastoral systems with traditional management in southeastern Mexico: a prototype of livestock agroforestry for cleaner production. Journal of Cleaner Production. 57:266 - 279. 
OFORI-BAH, A. y ASAFU-ADJAYE, J. 2011. Scope economies and technical efficiency of cocoa agroforesty systems in Ghana. Ecological Economics. 70(8):1508 - 1518.

OROZCO, L., VILLALOBOS, M., ORTÍZ, A., RIASCOS, L., MÉNDEZ, J. y SÁNCHEZ, V. 2008. Las fincas indígenas Bribri y Cabécar de Talamanca, Costa Rica. Agroforestería en las Américas. 46:14 - 20.

PAREDES, M. 2001. Identificación y caracterización de agroecosistemas de las riveras del Río Mejicano Municipio de Tumaco. Trabajo de grado Ingeniero Agroforestal. Facultad de Ciencias Agrícolas. Universidad de Nariño. Pasto 80 p.

PARRA, M. 1991. El agroecosistema: un concepto básico para entender el cambio tecnológico. p. 51-70. En Memorias 1er Simposio Nacional sobre Agricultura Sostenible: una opción para el desarrollo sin deterioro ambiental. Chapingo, México.

PE’DELAHORE, P. 2014. Farmers accumulation strategies and agroforestry systems intensification: the example of cocoa in the central region of Cameroon over the 1910-2010 period. Agroforestry Systems. 88(6):1157 - 1166.

PERDOMO, M. 2012. Caracterización de la cadena de abastecimiento y la cadena de valor del cacao en Colombia. Trabajo de grado Ingeniero Industrial. Facultad de Ingeniería. Pontificia Universidad Javeriana. Bogotá. 91 p.

PERZ, S. 2001. Household demographic factors as life cycle determinants of land use in the Amazon. Population Research and Policy Review. 20:159 - 18.

PORRO, R., LOPEZ-FELDMAN, A., VELA-ALVARADO, J., QUIÑONEZ-RUÍZ, L., SEIJAS-CARDENAS, Z., VÁSQUEZ-MACEDO, M. y CARDENASRUIZ, J. 2014. Forest use and agriculture in Ucayali, Peruvian Amazon: Interactions among livelihood strategies, income and environmental outcomes. Tropics. 23(2):47 - 62 .
PORRO, R., LOPEZ-FELDMAN, A. y VELAALVARADO, J. 2015. Forest use and agriculture in Ucayali, Peru: Livelihood strategies, poverty and wealth in an Amazon frontier. Forest Policy and Economics. 51:47 - 56.

PRECIADO, O., OCAMPO, C., y BALLESTEROS, W. 2011. Caracterización del sistema tradicional de producción de cacao (Theobroma cacao L.), en seis núcleos productivos del municipio de Tumaco, Nariño. Revista de Ciencias Agrícolas. 28(2):58 - 69.

QUAYE, A., HALL, C. y LUZADIS, V. 2010. Agricultural land use efficiency and food crop production in Ghana. Environment, Development and Sustainability. 12(6):967 - 983.

RUF, F. 2011. The myth of complex cocoa agroforests: the case of Ghana. Human Ecology. 39(3):373 - 388.

SÁENZ, Y. 2012. Aporte del cacaotal en la economía y nutrición familiar en Waslala, Nicaragua. Tesis de maestría. Magister Scientiae en Agroforestería Tropical. CATIE. Turrialba, Costa Rica. 118 p.

SÁNCHEZ, A. y AIDE, T. 2013. Consequences of the Armed Conflict, Forced Human Displacement, and Land Abandonment on Forest Cover Change in Colombia: A Multi-scaled Analysis. Ecosystems. 16(6):1052 - 1070.

SCHROTH, G. y RUF, F. 2014. Farmer strategies for tree crop diversification in the humid tropics. A review. Agronomy for Sustainable Development. 34(1):139 - 154.

SUPERINTENDENCIA DE INDUSTRIA Y COMERCIO (SIC). 2012. Cadena productiva del cacao: diagnóstico de libre competencia. En: SIC Colombia. http://www.sic.gov.co/drupal/sites/ default/files/files/Cacao.pdf. 6 p; consulta: Abril, 2015.

SOLANO, C., BERNUÉS A., ROJAS, F., JOAQUÍN, N., FERNÁNDEZ, W. y HERRERO, H. 2000. Relationships between management intensity and 
structural and social variables in dairy and dualpurpose systems in Santa Cruz, Bolivia. Agricultural Systems. 65(3):159 - 177.

SOMARRIBA, E., TRIVELATO, M., VILLALOBOS, M., SUÁREZ, A., BENAVIDES, P.; MORAN, K., OROZCO, L. y LÓPEZ, A. 2003. Diagnóstico agroforestal de pequeñas fincas cacaoteras orgánicas de indígenas Bribri y Cabécar de Talamanca, Costa Rica. Agroforestería en las Américas. 10(37-38):24 - 30.

SOMARRIBA, E., SUÁREZ-ISLAS, A., CALEROBORGE, W., VILLOTA, A., CASTILLO, C., VÍLCHEZ, S. y CERDA, R. 2014. Cocoa - timber agroforestry systems: Theobroma cacao - Cordia alliodora in Central America. Agroforestry Systems. 88(6):1001 - 1019.

SUÁREZ, J. 2009. Análisis de rentabilidad en los sistemas tradicionales de producción y la incorporación de los sistemas silvopastoriles en fincas de doble propósito, Matagalpa - Nicaragua. Tesis de maestría. Magister Scientiae en Agroforestería Tropical. CATIE. Turrialba, Costa Rica. 102 p.

TORUÑO, I. 2012. Análisis financiero-económico de fincas con varias actividades productivas y el rol de la familia en la producción y toma de decisiones en el Centro Norte de Nicaragua. Tesis de maestría. Magister Scientiae en Agroforestería Tropical. CATIE. Turrialba, Costa Rica. 158 p.

UBERHUAGA, P.; SMITH-HALL, C. y HELLES, F. 2012. Forest income and dependency in lowland Bolivia. Environment, Development and Sustainability. 14(1):3 - 23.

VAAST, P. y SOMARRIBA, E. 2014. Trade-offs between crop intensification and ecosystem services: the role of agroforestry in cocoa cultivation. Agroforestry Systems. 88(6):947 - 956.

VIGNERI, M. 2007. Drivers of cocoa production growth in Ghana. Overseas Development Institute Project Briefing. 4:4 p. 